\title{
Genetic Variability, Correlation and Path Analysis in Rice under Optimum and Stress Irrigation Regimes
}

\author{
Mina ABARSHAHR, Babak RABIEI*, Habibollah Samizadeh LAHIGI \\ University of Guilan, Faculty of Agricultural Sciences, Departament of Agronomy and \\ Plant Breeding, Iran; rabiei@guilan.ac.ir ('corresponding author)
}

\begin{abstract}
In order to estimate genetic variability and relationships among some agronomic traits of rice an experiment were conducted with 30 varieties of rice under two irrigation regimes. There were significant differences among the varieties for all traits. Broad-sense heritability varied from 0.05 for brown grain width to 0.99 for plant height and number of spikelet for panicle under optimum irrigation and from 0.1 for brown grain width to 0.99 for plant height. Evaluation of phenotypic and genotypic coefficient of variations (CV) showed that the lowest and highest phenotypic CV under optimum irrigation regime was observed to panicle fertility percentage and paddy yield and genotypic CV was related to brown grain width and plant height, respectively, while under drought stress condition, days to $50 \%$ flowering had the lowest phenotypic and genotypic CV and paddy yield and plant height had the highest phenotypic and genotypic CV. Furthermore, the lowest and highest expected genetic advance using selection intensity of $10 \%$ (i=1.75) were evaluated for brown grain width and plant height under optimum irrigation regime, respectively. Path analysis for paddy yield indicated that the number of spikelet per panicle and flag leaf length had positive direct effects and days to complete maturity and plant height had negative direct effects on paddy yield under optimum irrigation condition, while flag leaf width and number of filled grains per panicle had positive direct effects and days to $50 \%$ flowering had negative direct effect on paddy yield under drought stress condition.
\end{abstract}

Keywords: correlation coefficients, drought stress, genetic advance, paddy yield, path analysis, rice

\section{Introduction}

The periods of water shortage in soil or air often were happened during the life cycle of plants, even outside the drought and semi-drought regions. Plant responses to water deficit are complex including compromise changes or harmful effects. Drought conditions or water deficit stress in dry agroecosystems have a severe effect on rice production and they cause a meaningful decrease on its yield. As a result, increase of resistance to drought stress especially in rice, that is the main agricultural crops in Asia, is necessary (Singh, 2003; Widawsky and O'Toole, 1990).

In many Asian countries, rice production would be decreased due to drought stress. Plant breeding and introducing drought resistant varieties as well as improve of drought resistance mechanisms will be useful to solve this problem. In the present research it has been tried to prove that the yield of the number of rice cultivars is higher than the other cultivars under water stress. Plant varieties with high yield are generally dwarf and have high harvest index and in comparison with the other cultivars but if drought occurs during the late stages of growth, these cultivars will escape from drought stress and they will usually go flowering later than other cultivars. During the late season drought stress, some of the cultivars compared to others, are not able to maintain green leaves and increase their dry matter. So, the suitable cultivars for drought condi- tions have high yield potential and high harvest index. Drought resistance cultivars escape from severe drought stress, occurred at the end of the rain season, by early flowering mechanism (Jearakongman et al., 1995). Among agronomic practices, planting date is very important to escape from drought stress at flowering stage (Garrityt and O’Toole, 1994).

During the late drought stress, the capacity of assimilate transmission into seeds increases and this is a useful physiological phenomenon under drought stress conditions. It has been reported that, during drought stress, enough dry matter stores in the vegetative organs and also there are some variations among cultivars regarding dry matter accumulation under drought stress conditions and non-stress conditions. Exertion of drought stress at vegetative stage leads to increase of dry matter accumulation in sinks called remobilization. The amount of accumulated dry matter in green leaves is different from one cultivar to another cultivar. In addition, the amounts of assimilate transferring from stems and leaves to filling grains will increase parallel with drought stress, on the other hand, drought stress is effective on stabilizing of yield in those cultivars which have late leaf senescence during the period of grain filling (Kumar et al., 2006).

Drought stress during vegetative growth, flowering and terminal period of rice cultivation, can interrupt flo- 
ret initiation (which cause spikelet sterility) and grain filling, respectively (Botwright Acuna et al., 2008). On the other hand, it has been proposed that grain filling is closely linked to the whole-plant senescence process (Yang and Zhang, 2006). In some under-saturated soil moisture conditions, dry matter production and grain yield decreased significantly (Jun et al., 2000).

\section{Materials and methods}

The experiment was carried out under optimum irrigation regime and drought stress conditions in a research farm located in Faculty of Agricultural Sciences, University of Guilan, Guilan, Iran during 2007 (49 $36^{\prime} \mathrm{E}$ longitude, $37^{\circ} 16^{\prime} \mathrm{N}$ latitude and $7 \mathrm{~m}$ altitude). The yearly average precipitation was $298 \mathrm{~mm}$ also the mean annual temperature was $18.8^{\circ} \mathrm{C}$. The site of study belonged to the semi-Mediterranean region based on Iran Meteorological Organization divisions, with warm summer and mild winter. Although the average annual rainfall in this area is the extremely high, but distribution of rainfall is not generally coincided with rice growing stages, especially reproductive stages (from April to September). To run this experiment, 30 rice cultivars were provided from Rice Research Institute, Rasht, Guilan, Iran. The seeds were germinated and then were transplanted into the main land. Studied cultivars included 13 native varieties from Iran ('Binam', 'Champabodar', 'Hasansaraei', 'Domzard,' 'Domsorkh', 'Domsephid,' 'Domsiah', 'Deylamani, 'Salari', 'Sadri', 'Anbarboo', 'Gharib' and 'Hashemi'), 7 Iranian improved cultivars ('Bejar', 'Khazar', 'Dorfak', 'Sephidroud', 'Kadous', 'Neda' and 'Nemat'), 7 bred cultivars from IRRI ('IR24', 'IR28', 'IR30, 'IR36', 'IR50', 'IR60', 'IR64') and 3 upland cultivars from Brazil ('Araguaya'), United state ('New Bonnet') and India ('Vandana'). This experiment was performed under two different conditions (normal and drought stress). The experimental design was Randomized Complete Blocks with three replications. The plots were apart each other about two meters. In each experimental unit, there were 5 rows with distance of $25 \times 25 \mathrm{~cm}$ and distance of experimental transplant units from each other was 1 meter. After randomization of treatments, transplanting was performed as a single plant. All deep water irrigation operations were done until 20 days after transplanting, in the other word, until the full establishment of seedlings, and then drought stress was induced by irrigation with holding, while under normal conditions rice plants were irrigated completely until 10 days before harvesting. A deep drain at the depth of $40 \mathrm{~cm}$ and width $30 \mathrm{~cm}$, was created to help the exit of extra water from plots in order to prevent influence of water into drought stressed plots. To improve plant growth nitrogen fertilizer from urea source was applied in the amount of $150 \mathrm{~kg}$ per hectare; two-thirds part of urea was used during transplantation and one-third part during tillering stage. Moreover, phosphorus fertilizer from ammonium phosphate source was used in the amount of $100 \mathrm{~kg}$ per hectare during transplantation.
The fifteen traits were evaluated under both optimum and drought irrigation regimes, included plant height $(\mathrm{cm})$, panicle length $(\mathrm{cm})$, number of panicles/plant, flag leaf length $(\mathrm{cm})$, flag leaf width $(\mathrm{cm})$, brown grain length $(\mathrm{mm})$, brown grain width $(\mathrm{mm})$, days to $50 \%$ flowering, number of days to complete maturity, number of filled grains/panicle, number of spikelet/panicle, panicle fertility percentage, 1000-grain weight $(\mathrm{g})$, paddy yield $(\mathrm{kg}$ $\mathrm{ha}^{-1}$ ) and chlorophyll index (SPAD reading). Chlorophyll index was measured 76 days after transplanting. At first, data obtained from each irrigation regime were separately used to analysis of variance, then to assess the relationship among studied traits, genotypic and phenotypic correlation coefficients were determined. In order to demonstrate the importance of each trait, phenotypic correlation coefficients between paddy yield and other traits were separated to direct (path coefficient) and indirect affects using Dewey and Lu method (Dewey and Lu, 1959). Phenotypic and genotypic coefficients of variations $(\mathrm{CV})$ for each trait were estimated by equations 1 and 2:
1) $C V=\frac{\sqrt{V_{G}}}{\mu} \times 100$
2) $C V=\frac{\sqrt{V_{P}}}{\mu} \times 100$

Broad-sense heritability was calculated by equation 3 and genetic advance percentage was calculated by applying a selection intensity of 10 percentages $(i=1.75)$.

$$
\text { 3) } H_{b}=\frac{V_{G}}{V_{P}}
$$

All statistical analyses were carried out of using SAS Ver. 9.1 and SPSS Ver. 15 software.

\section{Results and discussion}

The results of analysis of variance showed that there were significant differences among varieties considering all studied traits under optimum irrigation regime and drought stress conditions $(p<0.01$, Tab. 1). Thus these significant differences among varieties showed high genetic diversity in studied populations, based on evaluated traits. Tab. 2 shows that the average of studied cultivars under both optimum irrigation regime and drought stress conditions calculated by HSD (Honestly Significant Difference or Tukey's test) and LSD (Least Significant Difference) at 5\% probability level. Estimation of paddy yield showed that, the best paddy yield was obtained from 'Nemat' cultivar under both optimum irrigation regime $\left(7430.4 \mathrm{~kg} \mathrm{ha}^{-1}\right)$ and drought stress conditions (5126.6 $\left.\mathrm{kg} \mathrm{ha}^{-1}\right)$, By contrast, the lowest paddy yield was achieved from 'Domsephid' cultivar under above mentioned conditions 2094.8 and $1565.9 \mathrm{~kg} \mathrm{ha}^{-1}$, respectively (Tab. 2). Also 
Tab. 1. Analysis of variance of studied traits in 30 rice cultivars under two irrigation regimes optimum irrigation $(\mathrm{O})$ and drought stress $(\mathrm{S})$

\begin{tabular}{|c|c|c|c|c|c|c|c|c|c|c|c|c|c|c|c|c|c|c|c|}
\hline \multicolumn{20}{|c|}{ Mean of squares (MS) } \\
\hline & & \multicolumn{2}{|c|}{$\begin{array}{l}\text { Plant } \\
\text { height }\end{array}$} & \multicolumn{2}{|c|}{$\begin{array}{l}\text { Panicle } \\
\text { length }\end{array}$} & \multicolumn{2}{|c|}{$\begin{array}{c}\text { Panicle number/ } \\
\text { plant }\end{array}$} & \multicolumn{2}{|c|}{ Flag leaf length } & \multicolumn{2}{|c|}{ Flag leaf width } & \multicolumn{2}{|c|}{$\begin{array}{l}\text { Brown grain } \\
\text { length }\end{array}$} & \multicolumn{2}{|c|}{$\begin{array}{l}\text { Brown grain } \\
\text { width }\end{array}$} & \multicolumn{2}{|c|}{$\begin{array}{c}\text { Days to } \\
50 \% \text { flowering }\end{array}$} & \multicolumn{2}{|c|}{$\begin{array}{l}\text { Days to complete } \\
\text { maturity }\end{array}$} \\
\hline S.O.V & $\mathrm{d}_{\mathrm{f}}$ & $\mathrm{O}$ & $S$ & $\mathrm{O}$ & $S$ & $\mathrm{O}$ & $S$ & $\mathrm{O}$ & $S$ & $\mathrm{O}$ & $S$ & $\mathrm{O}$ & $S$ & $\mathrm{O}$ & $S$ & $\mathrm{O}$ & $S$ & $\mathrm{O}$ & $S$ \\
\hline Block & 2 & $0.11^{\mathrm{ns}}$ & $0.96^{\mathrm{ns}}$ & $0.74^{\mathrm{ns}}$ & $0.63^{\mathrm{ns}}$ & $0.58^{\text {ns }}$ & $1.51^{\mathrm{ns}}$ & $12.34^{\circ}$ & $4.65^{\mathrm{ns}}$ & $0.01^{\text {ns }}$ & $0.34^{\prime \prime}$ & $0.0007^{\mathrm{ns}}$ & $0.02^{\mathrm{ns}}$ & $0.02^{\text {ns }}$ & $0.0003^{\text {ns }}$ & $1.63^{\mathrm{ns}}$ & $3.24^{\mathrm{ns}}$ & $7.24^{\mathrm{ns}}$ & $15.01^{\text {ns }}$ \\
\hline Genotype & 29 & $3030.41^{*}$ & $3094.49^{*}$ & $15.62^{*}$ & $33.008^{*}$ & $79.36^{\prime \prime}$ & $50.55^{*}$ & $49.08^{* *}$ & $49.23^{*}$ & $0.26^{\circ}$ & $0.18^{* *}$ & $1.21^{*}$ & $1.33^{*}$ & $0.02^{*}$ & $0.003^{*}$ & $51.56^{*}$ & $40.36^{*}$ & $113.52^{*}$ & $75.5^{*}$ \\
\hline Error & 58 & 1.49 & 1.5 & 0.56 & 0.3 & 1.12 & 0.73 & 3.09 & 3.5 & 0.02 & 0.03 & 0.002 & 0.01 & 0.01 & 0.0004 & 1.95 & 5.92 & 11.95 & 14.22 \\
\hline CV (\%) & & 1.98 & 1.33 & 2.37 & 1.94 & 4.89 & 5.16 & 4.94 & 6.11 & 9.62 & 11.23 & 0.7 & 1.61 & 6.39 & 1.25 & 1.6 & 2.92 & 3.15 & 3.61 \\
\hline
\end{tabular}

ns: non-significant ${ }^{*}$ and ${ }^{* *}$ : significant at $5 \%$ and $1 \%$ probability levels, respectively

Tab. 1. Analysis of variance of studied traits in 30 rice cultivars under two irrigation regimes optimum irrigation $(\mathrm{O})$ and drought stress(S)-Continous

\begin{tabular}{|c|c|c|c|c|c|c|c|c|c|c|c|c|c|}
\hline \multicolumn{14}{|c|}{ Mean of squares (MS) } \\
\hline & & \multicolumn{2}{|c|}{$\begin{array}{l}\text { Number of filled } \\
\text { grains/panicle }\end{array}$} & \multicolumn{2}{|c|}{$\begin{array}{c}\text { Number of spikelet } \\
\text { per panicle }\end{array}$} & \multicolumn{2}{|c|}{ Panicle fertility percentage } & \multicolumn{2}{|c|}{ 1000-grain weight } & \multicolumn{2}{|c|}{ Paddy yield } & \multicolumn{2}{|c|}{ Chlorophyll index } \\
\hline S.O.V & $\mathrm{d}_{f}$ & $\mathrm{O}$ & $S$ & $\mathrm{O}$ & S & $\mathrm{O}$ & $S$ & $\mathrm{O}$ & S & $\mathrm{O}$ & $S$ & $\mathrm{O}$ & S \\
\hline Block & 2 & $16 / 87^{\circ}$ & $6 / 21^{\text {ns }}$ & $19.3^{\circ}$ & $1.47^{\mathrm{ns}}$ & $0.14^{\mathrm{ns}}$ & $1.65^{\mathrm{ns}}$ & $20.6^{6^{*}}$ & 35.6 & $1656063^{4 \mathrm{~ns}}$ & $459119.4^{\mathrm{ns}}$ & $6.61^{\mathrm{ns}}$ & $92.05^{\prime \prime}$ \\
\hline Genotype & 29 & $1271 / 38^{* \prime}$ & $1136 / 56$ & $1475.65^{*}$ & $1115.73^{*}$ & $16.66^{\circ}$ & $51.29^{*}$ & $16.8^{7^{*}}$ & $21.85^{*}$ & $4048717.1^{*}$ & $2035926.49^{\prime \prime}$ & $21.1^{*}$ & $18.52^{*}$ \\
\hline Error & 58 & $5 / 38$ & $3 / 03$ & 4.92 & 2.05 & 1.82 & 1.95 & 3.01 & 4.98 & 874308.8 & 250918.88 & 4.47 & 8.19 \\
\hline $\mathrm{CV}(\%)$ & & $1 / 43$ & $1 / 5$ & 1.26 & 0.97 & 1.45 & 1.78 & 5.55 & 7.4 & 19.36 & 16.7 & 5.46 & 7.82 \\
\hline
\end{tabular}

ns: non-significant ${ }^{*}$ and ${ }^{* *}$ : significant at $5 \%$ and $1 \%$ probability levels, respectively 
Tab. 2. Mean of 30 rice varieties for measured traits under two irrigation regimes, optimum irrigation $(\mathrm{O})$ and drought stress $(\mathrm{S})$

\begin{tabular}{|c|c|c|c|c|c|c|c|c|c|c|c|c|c|c|c|c|c|c|c|c|c|c|c|c|c|c|c|c|c|c|}
\hline \multirow{2}{*}{ riety } & \multicolumn{2}{|c|}{ PH } & \multicolumn{2}{|c|}{ PL } & \multicolumn{2}{|c|}{ PanN } & \multicolumn{2}{|c|}{ FLL } & \multicolumn{2}{|c|}{ FLW } & \multicolumn{2}{|c|}{ BGL } & \multicolumn{2}{|c|}{ BGW } & \multicolumn{2}{|c|}{ DF } & \multicolumn{2}{|c|}{ DM } & \multicolumn{2}{|c|}{ NFG } & \multicolumn{2}{|c|}{ NSP } & \multicolumn{2}{|c|}{ PFP } & \multicolumn{2}{|c|}{ 100-GW } & \multicolumn{2}{|c|}{ PY } & \multicolumn{2}{|c|}{ CI } \\
\hline & $\mathrm{O}$ & $S$ & $\mathrm{O}$ & $S$ & $\mathrm{O}$ & $S$ & $\mathrm{O}$ & $\mathrm{S}$ & $\mathrm{O}$ & $S$ & $\mathrm{O}$ & $S$ & $\mathrm{O}$ & $S$ & $\mathrm{O}$ & S & $\mathrm{O}$ & $S$ & $\mathrm{O}$ & $S$ & $\mathrm{O}$ & & $\mathrm{O}$ & s & $\mathrm{O}$ & $S$ & $\mathrm{O}$ & s & & $S$ \\
\hline am' & 2.5 & & & & & & & & 69 & 1.52 & & 6.89 & 71 & 1.6 & & & & & & & 169.33 & & & & 58 & & & & & 5.8 \\
\hline & & & & & & & & & & & & & & & & & & & & & & & & & & & & & & 5.9 \\
\hline & & & & & & & & & & & & & & & & & & & & 2.33 & 4.66 & 66 & 3.96 & & 05 & 20 & & & 5.26 & 4.6 \\
\hline & 13 & כ. & 14 & & 86 & & & & & 1.00 & & & & & & & & & & & 8.66 & 6.66 & 4.54 & & 0.88 & 55 & 2.25 & & .56 & 4.43 \\
\hline 'Domsorkh' & .53 & .38 & .37 & 94 & 26 & 1.53 & 34.68 & 5.7 & 1.46 & 1.21 & & 7.46 & 1.71 & 1.63 & 66 & & 118 & 103.66 & 0.66 & 7.33 & 1) & 123.66 & 4.76 & 76.84 & 9.95 & .47 & 223.91 & 109.66 & 6.16 & 5.26 \\
\hline 'Domsephi & 16 & & & & 63 & & & & 2 & 1.12 & & & & 1.33 & 33 & 91.33 & 122 & 111.33 & & 1.33 & 140.33 & 121.66 & & 61 & 5.54 & 97 & & 65.89 & 46 & 33 \\
\hline & 98 & 132. & 28.34 & 25.66 & 18.66 & & & 78 & 4 & & & & & 1.6 & 66 & & 111.66 & 97 & & 33 & & 163 & & & 05 & 15 & & & 5.5 & 9.9 \\
\hline 'Deyla & & & & 24.76 & 06 & & & & & 1.1 & & & & 1. & 84 & & & & & & & 9.33 & & & 34 & & & & 86 & 3.4 \\
\hline & 15 & & & 26.8 & 16.26 & 13.7 & & 62 & 42 & 1.3 & 2 & 4 & & & 93 & & 10 & 98 & 66 & 98 & 2 & .66 & 31 & & 57 & 35 & 3.96 & 77.46 & 7.36 & 23 \\
\hline & & 139.1 & & 5.13 & & 15. & & & & & & & & & 90 & & & & & 101.33 & 154. & & & & & & & & 5 & 76 \\
\hline 'Anbarboo' & & 148.1 & & 27.13 & 96 & & & 63 & & & & & & & 88.33 & & & & & 66 & & & & & & 54 & & & 63 & .7 \\
\hline ‘G & & 146. & & 28.03 & 14.86 & & & & & & & & & & & & & & & & & & & & & & & & & 23 \\
\hline 'Hashemi' & .03 & & & & 22.3 & & & 30. & & 1.49 & & & & 1. & 33 & & & 86 & & 99 & & 144.66 & & & & 43 & & & 8 & 06 \\
\hline & & 100.9 & & 94 & 22.42 & 21.86 & 29.94 & 28.48 & & 1.7 & 4 & & & 1. & 33 & & & & & & & 162 & & & & & & & 13 & 5.2 \\
\hline & 18 & 114.88 & 31.87 & 25.53 & 14.03 & 11.53 & 32.76 & 26.58 & 34 & 1.78 & 2 & 7.29 & & & 66 & & 3.33 & 106.33 & 170.66 & 106.33 & & 131 & & & & 55 & & & 16 & 13 \\
\hline & & & 33.86 & 30.8 & 86 & 15 & & & & 1. & & & & & & & & & & 104.66 & & 135.66 & & & & & & & 26 & 53 \\
\hline 'Sephidroud' & & 89.62 & 34.1 & & .56 & 24.2 & 35.52 & 35.24 & 95 & 1.91 & 6 & 35 & 99 & 1.76 & .66 & 33 & 107 & & 200.66 & & 213.66 & 171 & 93.95 & & 1.28 & .41 & 86.95 & & 38.36 & 26 \\
\hline & & 94.3 & 35 & 73 & 13 & 18.63 & 35 & 33.9 & 68 & 1.8 & & 7.77 & 76 & 1.72 & 33 & 81.66 & 107.66 & 103 & & 103 & 189 & 165 & 93.89 & 97 & 2.87 & 2.6 & 5.28 & 2.09 & 3.4 & 1.13 \\
\hline & & 94.5 & 33.39 & 26.92 & 96 & 18 & 36.02 & 31.55 & 1.67 & 1.68 & & 7.59 & 35 & 1.71 & 3.66 & $8 c$ & 109.33 & 104.33 & 184 & 104.33 & 1.33 & 152.66 & 91.39 & 31 & 32.31 & 91 & 2.91 & 424.02 & .7 & 36 \\
\hline 'Nemat' & .78 & 98.34 & 35. & 35.2 & 31.16 & & 2 & 37.4 & 4 & 1.9 & & 67 & 72 & 1.69 & 89.33 & 8 & & 95.33 & 209.33 & 104 & 1.66 & 211.33 & 94.43 & 91.48 & & 08 & 30.37 & & 39 & 7.56 \\
\hline & & 76.26 & & 30.26 & 26.4 & 18.83 & 29.32 & 27.22 & 49 & 1.36 & & 6.79 & 75 & 1.69 & 93 & 8 & 122 & 115.66 & 161 & 116.66 & 9.33 & 148.33 & 89.77 & 82.7 & 4 & 11 & & & 39.16 & 3.6 \\
\hline 'IR28' & & 74.8 & 31.97 & 28.7 & 26.23 & 17.03 & 39.42 & 34.77 & 1.76 & 1.7 & 7 & 6.13 & 1.74 & 1.66 & 90 & 80 & 2.33 & 98 & 6.66 & 98 & 6.66 & 147.66 & 89.29 & 81.03 & 4.32 & 19 & 50.01 & 958.002 & 9.8 & 2.93 \\
\hline 'IR30’ & 57 & 87.04 & 31.53 & & 27.5 & 15.13 & 38.87 & 29.1 & 1.71 & 1.42 & 2 & 04 & 63 & 1.65 & 85 & 82.33 & 107.33 & 107.33 & 2.33 & 100 & 33 & 130 & 89.14 & 76.41 & 33.98 & .11 & 40.71 & 88.27 & 9.93 & .36 \\
\hline IR36 & & 83.9 & 30.53 & 29.16 & .53 & 15.66 & 28.94 & 28.82 & 1.5 & 1.52 & 79 & 6.77 & 1.71 & 1.57 & 80 & 75.66 & 103 & 99.66 & 3.33 & 99.66 & 7.33 & 125 & 91.63 & 76.8 & 30.76 & .59 & 158.12 & 32952 & .46 & 6.33 \\
\hline 'IR50’ & 23 & 79.66 & 31.91 & 27.76 & 25.66 & 23.5 & 37.98 & 29.44 & 1.68 & 1.56 & 7.51 & 7.44 & 1.99 & 1.66 & 85 & 84 & 105.33 & 107.66 & 1.66 & 102.66 & 181.66 & 169.66 & 89.005 & 75.64 & 33.69 & .15 & 931.87 & 3179.72 & 1.5 & 4.76 \\
\hline 'IR60' & 26 & 78.57 & 30.53 & 28.96 & 25.56 & 17.96 & 33.06 & 22.68 & 1.64 & 1.31 & 6.98 & 6.94 & 1.74 & 1.72 & 86 & 84.66 & 108 & 4.66 & 6.33 & 104.66 & 6.66 & 148.66 & 93.8 & 81.16 & 28.8 & .43 & 70.1 & 2295.87 & 2.4 & 8.8 \\
\hline IR64' & 63 & 84.83 & 31.8 & 27.9 & 28.76 & 22.5 & 33.59 & 32.37 & 1.66 & 1.64 & 7.32 & 7.28 & 1.74 & 1.7 & 88 & 80.66 & 109.66 & 107.66 & 173.33 & 107.66 & 200.33 & 162 & 86.51 & 79.62 & 31.9 & 30.36 & 5499.23 & 3367.76 & 2.9 & 33.83 \\
\hline guaya' & .71 & 119.63 & 27.35 & 22.3 & 16.26 & 12.3 & 32.64 & 32.39 & 1.61 & 1.66 & 7.11 & 7.08 & 1.72 & 1.71 & 86.33 & 87 & 120.66 & 116.66 & 162 & 115.66 & 172.33 & 125 & 93.98 & 77.61 & 30.87 & 29.71 & 5155.76 & 3396.52 & 3.2 & 36.36 \\
\hline & & & & 23.4 & & & & 31.96 & & 1.4 & & 7.14 & & 1.63 & & 80 & & & & 103.66 & & & 92.18 & 67.6 & & & & & 44.03 & 37.33 \\
\hline & 134.22 & 119.8 & & & 17.6 & & & & & 1.6 & & 6. & & 1. & & & & 101. & 145. & 101. & 159.33 & 154.66 & & & & & & & 3 & 76 \\
\hline & & & & 1.7 & 3.4 & 2.76 & 5.6 & 6.02 & 0.5 & 0.5 & & 0.3 & 0.3 & 0.0 & 4.5 & & 11.13 & 12.1 & & & & & & & & & & 12.5 & 8.8 & 21 \\
\hline $\mathrm{LSD}_{0.05}$ & 1.99 & & 1.22 & 0.9 & 1.73 & 1.4 & 2.87 & 3.06 & 0.25 & 0.28 & & 0.18 & 0.18 & 0.03 & 2.28 & 3.97 & 5.65 & 6.16 & 3.19 & 2.84 & 3.62 & 2.01 & 2.2 & 2.28 & 2.83 & 3.65 & 1528.2 & 818.7 & 3.45 & 4.67 \\
\hline
\end{tabular}

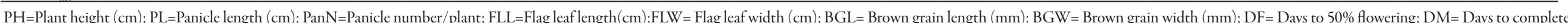

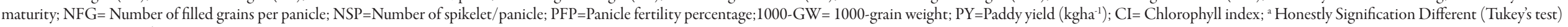

at $5 \%$ probability level; ${ }^{b}$ Least Significant Different at $5 \%$ probability level 
138

the result indicated that effect of year was not significant on evaluated traits, nonetheless the optimal and high yield components have made 'Nemat' cultivar as a high paddy yield during two experimental years. The 'Nemat' cultivar showed high phenotypic value under both irrigation regimes in respect of many important aspects of agronomic traits or related traits to yield. In case of panicle length, panicles number per plant, brown grain length, number of filled grains per panicle, number of spikelet per panicle, 1000-grains weight and paddy yield under both irrigation regimes and also panicle fertility percentage under drought stress conditions (most traits in path analysis were determined as the most important traits affecting on paddy yield) 'Nemat' cultivars was superior cultivar compared with other studied cultivars, so it can be considered as one of the most important cultivars. The obtained results from genotype variation coefficient demonstrated that, plant height $(25.55$ percent $)$, panicle number per plant ( 23.58 percent) and paddy yield (21.30 percent) had maximum amount and browning grain width (1.81 percent) had the lowest amount (Tab. 3). Also under drought stress conditions plant height (27.14 percent), paddy yield (25.73 percent) and panicle number per plant (24.46 percent) had maximum amount. Furthermore, number of days to $50 \%$ flowering (4.07 percent) and number of day to complete maturity ( 4.33 percent) had the lowest amount of coefficient of genotype variation (Tab. 4).

The high variation in plant height and paddy yield especially under drought stress conditions gives promising news about applying these traits in accordance with the goals of breeding program. Evaluation of genetic advance on plant height, panicles number per plant and paddy yield showed that under both optimum irrigation regime and drought stress conditions these traits had the maximum increase (Tab. 3 and Tab. 4). Similar to variation coefficient, when variability in the studied population is high, an effective selection would be possible for breeding of most traits, so that high amount of genetic advance would be confirmed. At the first stage, it has been used the simple selection method to improve these traits and then increase favorite genes sharing with addition effects on the population and after that it has been used full capacity of gene with this kind of effect, thus it has been possible to use the other effects of genes such as dominance and super dominance and presumably heterosis phenomenon. Two traits including brown grain width (0.57) and panicle fertility percentage (3.43) under optimum irrigation condition, and chlorophyll index (4.82) under stress conditions had the lowest average of genetic advance percentage (Tab. 3 and Tab. 4). Upon broad-sense heritability calculating for traits, all of the traits except for brown grain width $(0.05)$ (Tab. 3) in optimum irrigation condition and all traits except brown grain width (0.1) and chlorophyll index (0.29) under drought stress conditions had broadsense heritability more than 50\% (Tab. 4). Mixing genotype cross effect $\times$ environment due to survey of experiment in one year could be one of the important reasons being traits high heritability in both experimental environments. The obtained results from broad-sense heritability of traits demonstrate that, plant height, number of spikelet per panicle and number of filled grains per panicle were affected by optimum irrigation regime and drought stress conditions. Brown grain width had broad-sense heritability (0.05) under optimum irrigation regime that shows the full impact of environment and crops conditions on this trait. In addition, under drought stress conditions brown grain width had broad-sense heritability $(0.1)$ that shows it had the same environment and genetic effects on this trait. Kumar et al. (2007) expressed that, the heritability of rice under drought stress is lower than the optimal irrigation. Ekanayake et al. (1985) believed that, avoiding drought stress in rice is dependent on tensile resistance of rice root system and concluded that, the root tensile resistance will have low heritability (39\%-47\%). Increasing the root tensile resistance and then drought resistance in order for the selection on base of line average will be better than individual selection. Phenotypic and genotypic correlation coefficients of studied traits under both optimum irrigation regime and drought conditions are presented in Tab. 5 and 6, respectively. Phenotypic correlations in terms of optimum irrigation regime showed that, there was significant correlation among paddy yield with plant height, panicle number per plant, flag leaf width, number of filled grains per panicle, number of spikelet per panicle and 1000 -grain weight $(p<0.01)$, panicle length, brown grain length a day to complete maturity and chlorophyll index $(p<0.05)$ (Tab. 5). All characteristics, except for plant height and number of days to complete maturity had positive correlation with final yield and these results were consistent with findings of Mehetre et al. (1994) and Lanceras et al. (2004). Significant and positive correlation between yield and other traits showed that each factor leads to changes in these traits will change correlation coefficients. It has been reported that, there is positive and significant correlation between panicle length and yield (Abd El Samie and Hasan, 1994; Bapo and Soundarapandian, 1992; Choudhury and Das, 1998; Padmavathi et al., 1996). Phenotypic correlations under drought stress conditions showed that, paddy yield had significant and positive correlation with plant height, panicle length, number of panicles per plant, flag leaf width, days to $50 \%$ flowering, number of filled grains per panicle, number of spikelet per panicle, 1000-grain weight ( $p<0.01)$, flag leaf length, brown grain width, panicle fertility percentage and chlorophyll index $(p<0.05)$ while correlation between paddy yield and plant height or paddy yield and days to $50 \%$ flowering were negative (Tab. 5). Garrity and O'Toole (1994) have been reported that, there is positive correlation between panicle fertility percentage and paddy yield under water stress conditions. In addition, Lafitte et al. (2006) showed that, there is a negative and significant correlation between plant heights and final yield under 
Tab. 3. Estimation of statistical and genetic parameters of studied traits under optimum irrigation regime

\begin{tabular}{|c|c|c|c|c|c|c|c|c|c|c|c|}
\hline & \multirow{2}{*}{ Min. } & \multirow{2}{*}{ Max. } & \multirow{2}{*}{ Range } & \multirow{2}{*}{ Mean $\pm S_{x}$} & \multicolumn{2}{|c|}{$\begin{array}{c}\text { Coefficient of } \\
\text { Variation (CV \%) }\end{array}$} & \multicolumn{3}{|c|}{ Variance components } & \multirow{2}{*}{ Heritability } & \multirow{2}{*}{$\begin{array}{c}\text { Geneti } \\
\text { advance } \\
(\%)^{\alpha}\end{array}$} \\
\hline & & & & & Genotypic & Phenotypic & $\begin{array}{c}\text { E.nviromental } \\
\text { Variance }\end{array}$ & $\begin{array}{c}\text { Genotypic } \\
\text { Variance }\end{array}$ & $\begin{array}{c}\text { Phenotypic } \\
\text { Variance }\end{array}$ & & \\
\hline $\mathrm{PH}$ & 72.83 & 174.16 & 101.33 & $124.35 \pm 31.78$ & 25.55 & 25.57 & 1.49 & 1009.64 & 1011.14 & 0.99 & 44.68 \\
\hline PL & 27.35 & 35.86 & 8.51 & $31.65 \pm 2.28$ & 7.07 & 7.46 & 0.56 & 5.02 & 5.58 & 0.89 & 11.74 \\
\hline PanN & 14.03 & 31.16 & 17.13 & $21.65 \pm 5.14$ & 23.58 & 24.08 & 1.12 & 26.08 & 27.21 & 0.95 & 40.40 \\
\hline FLL & 28.94 & 43.35 & 14.41 & $35.59 \pm 4.04$ & 11.001 & 12.05 & 3.09 & 15.33 & 18.42 & 0.83 & 17.56 \\
\hline FLW & 1.22 & 2.69 & 1.46 & $1.64 \pm 0.29$ & 17.17 & 20.13 & 0.02 & 0.08 & 0.11 & 0.72 & 25.62 \\
\hline BGL & 6.06 & 8.76 & 2.69 & $7.37 \pm 0.63$ & 8.57 & 8.68 & 0.002 & 0.4 & 0.41 & 0.97 & 14.82 \\
\hline BGW & 1.62 & 1.99 & 0.37 & $1.74 \pm 0.08$ & 1.81 & 8.11 & 0.01 & 0.001 & 0.02 & 0.05 & 0.57 \\
\hline DF & 80 & 95.33 & 15.33 & $87.33 \pm 4.14$ & 4.65 & 4.92 & 1.95 & 16.54 & 18.49 & 0.89 & 7.70 \\
\hline $\mathrm{DM}$ & 101 & 122 & 21 & $109.65 \pm 6.15$ & 5.30 & 6.17 & 11.95 & 33.86 & 45.81 & 0.73 & 7.98 \\
\hline NFGe & 127.66 & 209.33 & 81.66 & $161.55 \pm 20.58$ & 12.71 & 12.79 & 5.38 & 422 & 427.38 & 0.98 & 22.11 \\
\hline NSP & 140 & 221.66 & 81.66 & $174.66 \pm 22.17$ & 12.67 & 12.73 & 4.92 & 490.24 & 495.17 & 0.99 & 22.07 \\
\hline PFP & 86.51 & 96.8 & 10.28 & $92.52 \pm 2.28$ & 2.32 & 2.74 & 1.82 & 4.61 & 6.44 & 0.71 & 3.43 \\
\hline 1000-GW & 25.54 & 35.6 & 10.05 & $31.23 \pm 2.37$ & 6.88 & 8.84 & 3.01 & 4.62 & 7.64 & 0.6 & 9.36 \\
\hline PY & 2094.78 & 7430.37 & 5335.59 & $4829.27 \pm 1161.71$ & 21.30 & 28.78 & 874308.8 & 1058136 & 1932445 & 0.54 & 27.58 \\
\hline $\mathrm{CI}$ & 34.4 & 44.3 & 9.9 & $38.7 \pm 2.65$ & 6.08 & 8.17 & 4.47 & 5.55 & 10.02 & 0.55 & 7.92 \\
\hline
\end{tabular}

PH=Plant height (cm); PL=Panicle length (cm); PanN=Panicle number/plant; FLL=Flag leaf length $(\mathrm{cm}) ; F L W=$ Flag leaf width (cm); BGL= Brown grain length (mm); $\mathrm{BGW}=$ Brown grain width (mm); DF= Days to $50 \%$ flowering; DM= Days to complete maturity; NFG= Number of filled grains per panicle; NSP=Number of spikelet/panicle; $\mathrm{PFP}=$ Panicle fertility percentage; $1000-\mathrm{GW}=1000$-grain weight; $\mathrm{PY}=$ Paddy yield $\left(\mathrm{kgha}^{-1}\right) ; \mathrm{CI}=\mathrm{Chlorophyll}$ index; ${ }^{\mathrm{a}} \mathrm{Honestly}$ Signification Different (Tukey's test) at $5 \%$ probability level; ${ }^{\mathrm{b}}$ Least Significant Different at $5 \%$ probability level; ${ }^{\alpha}$ Genetic advance with $10 \%$ selection intensity $(\mathrm{i}=1.75)$

Tab. 4. Estimation of statistical and genetic parameters of studied traits under drought stress condition

\begin{tabular}{|c|c|c|c|c|c|c|c|c|c|c|c|}
\hline & \multirow{2}{*}{ Min } & \multirow{2}{*}{ Ma. } & \multirow{2}{*}{ Range } & \multirow{2}{*}{ Mean $\pm S_{X}$} & \multicolumn{2}{|c|}{$\begin{array}{c}\text { Coefficient of } \\
\text { Variation }(\mathrm{CV} \%)\end{array}$} & \multicolumn{3}{|c|}{ Variance components } & \multirow{2}{*}{ Heritability } & \multirow{2}{*}{$\begin{array}{c}\text { Genetic } \\
\text { advance } \\
(\%)^{\alpha}\end{array}$} \\
\hline & & & & & Genotypic & Phenotypic & $\begin{array}{c}\text { Environmental } \\
\text { Variance }\end{array}$ & $\begin{array}{l}\text { Genotypic } \\
\text { Variance }\end{array}$ & $\begin{array}{c}\text { Phenotypic } \\
\text { Variance }\end{array}$ & & \\
\hline $\mathrm{PH}$ & 71.8 & 172.25 & 100.44 & $118.3 \pm 32.11$ & 27.14 & 27.16 & 1.5 & 1031 & 1032.51 & 0.99 & 47.46 \\
\hline PL & 22.3 & 35.2 & 12.89 & $28.42 \pm 3.31$ & 11.61 & 11.77 & 0.3 & 10.9 & 11.21 & 0.97 & 20.04 \\
\hline PanN & 11.53 & 28.2 & 16.66 & $16.65 \pm 4.1$ & 24.46 & 25.004 & 0.73 & 16.61 & 17.35 & 0.95 & 41.89 \\
\hline FLL & 22.68 & 41.92 & 19.24 & $30.63 \pm 4.05$ & 12.74 & 14.13 & 3.5 & 15.24 & 18.75 & 0.81 & 20.10 \\
\hline FLW & 1.12 & 2.18 & 1.05 & $1.53 \pm 0.24$ & 14.53 & 18.38 & 0.03 & 0.05 & 0.08 & 0.62 & 20.10 \\
\hline BGL & 6.03 & 8.67 & 2.63 & $7.06 \pm 0.66$ & 9.39 & 9.49 & 0.01 & 0.44 & 0.45 & 0.97 & 16.25 \\
\hline BGW & 1.33 & 1.84 & 0.51 & $1.66 \pm 0.1$ & 6.004 & 6.004 & 0.001 & 0.01 & 0.01 & 0.1 & 10.50 \\
\hline DF & 75.66 & 91.33 & 15.66 & $83.07 \pm 3.66$ & 4.07 & 5.02 & 5.92 & 11.48 & 17.4 & 0.65 & 5.79 \\
\hline $\mathrm{DM}$ & 96.66 & 116.66 & 20 & $104.21 \pm 5.01$ & 4.33 & 5.64 & 14.22 & 20.43 & 34.65 & 0.58 & 5.82 \\
\hline NFGe & 92 & 193.33 & 101.33 & $115.85 \pm 19.46$ & 16.77 & 16.84 & 3.03 & 377.84 & 380.88 & 0.99 & 29.24 \\
\hline NSP & 121.66 & 211.33 & 89.66 & $147.52 \pm 19.28$ & 13.06 & 13.09 & 2.05 & 371.23 & 373.28 & 0.99 & 22.79 \\
\hline PFP & 67.6 & 91.48 & 23.87 & $78.32 \pm 4.13$ & 5.17 & 5.47 & 1.95 & 16.45 & 18.4 & 0.89 & 8.56 \\
\hline 1000-GW & 23.97 & 35.08 & 11.11 & $30.16 \pm 2.69$ & 7.85 & 10.79 & 4.98 & 5.62 & 10.61 & 0.52 & 10.01 \\
\hline PY & 1565.89 & 5126.62 & 3560.73 & $2997.81 \pm 823.79$ & 25.73 & 30.68 & 250918.88 & 595002.5 & 845921.4 & 0.7 & 37.76 \\
\hline $\mathrm{CI}$ & 32.33 & 42.93 & 10.6 & $36.59 \pm 2.48$ & 5.06 & 9.32 & 8.19 & 3.44 & 11.64 & 0.29 & 4.82 \\
\hline
\end{tabular}

PH=Plant height (cm); PL=Panicle length (cm); PanN=Panicle number/plant; FLL=Flag leaf length $(\mathrm{cm}) ; F L W=$ Flag leaf width (cm); BGL= Brown grain length (mm); $\mathrm{BGW}=$ Brown grain width $(\mathrm{mm}) ; \mathrm{DF}=$ Days to $50 \%$ flowering; $\mathrm{DM}=$ Days to complete maturity; NFG= Number of filled grains per panicle; NSP=Number of spikelet/panicle; PFP=Panicle fertility percentage; $1000-\mathrm{GW}=1000$-grain weight; PY=Paddy yield $\left(\mathrm{kgha}^{-1}\right) ; \mathrm{CI}=\mathrm{Chlorophyll}$ index; ${ }^{\mathrm{a}} \mathrm{Honestly}$ Signification Different (Tukey's test) at $5 \%$ probability level; ${ }^{b}$ Least Significant Different at $5 \%$ probability level; ${ }^{\alpha}$ Genetic advance with $10 \%$ selection intensity $(\mathrm{i}=1.75)$

drought stress conditions. The significant and positive correlation was observed between flag leaf width and paddy yield while under conditions of optimum irrigation regime, number of spikelet per panicle had significant and positive correlation with paddy yield that shows the importance of flag leaf at grain filling stage under water deficit stress. Genotype correlation under optimum irrigation conditions showed that, there is a significant and positive correlation among paddy yield and panicle length, panicle number per plant, flag leaf width, brown grain length, the number of filled grain per panicle, number of spikelet per panicle, 1000-grain weight and chlorophyll index while paddy yield had significant and negative correlation with days to complete maturity and plant height (Tab. 6). Gen- 
140

Tab. 5. Phenotypic correlation coefficients between traits in 30 rice cultivars in two conditions, optimum irrigation (up) and drought stress (down)

\begin{tabular}{|c|c|c|c|c|c|c|c|c|c|c|c|c|c|c|c|}
\hline Trait & 1 & 2 & 3 & 4 & 5 & 6 & 7 & 8 & 9 & 10 & 11 & 12 & 13 & 14 & 15 \\
\hline 1 & 1 & -0.14 & $-0.71^{\prime \prime}$ & 0.32 & -0.17 & $-0.44^{\circ}$ & -0.1 & 0.14 & 0.29 & $-0.51^{*}$ & $-0.61^{*}$ & $0.46^{\circ}$ & -0.28 & $-0.66^{\circ}$ & $-0.63^{\prime \prime}$ \\
\hline 2 & -0.12 & 1 & $0.5^{\prime \prime}$ & 0.17 & $0.5^{*}$ & 0.22 & 0.32 & -0.2 & -0.18 & $0.57^{\prime \prime}$ & $0.54^{\prime \prime}$ & 0.15 & $-0.39^{\circ}$ & $0.45^{\circ}$ & -0.26 \\
\hline 3 & -0.46 & $0.63^{\prime \prime}$ & 1 & 0.01 & $0.39^{\circ}$ & $0.46^{\circ}$ & 0.19 & -0.24 & -0.3 & $0.63^{*}$ & $0.67^{\prime \prime}$ & -0.18 & $0.43^{\circ}$ & $0.69^{*}$ & 0.32 \\
\hline 4 & 0.1 & 0.21 & 0.25 & 1 & 0.21 & -0.04 & -0.08 & 0.32 & 0.16 & 0.03 & 0.03 & 0.03 & 0.27 & 0.18 & 0.2 \\
\hline 5 & -0.25 & $0.45^{\circ}$ & $0.52^{\circ}$ & 0.35 & 1 & 0.06 & $0.37^{\circ}$ & -0.26 & -0.07 & $0.77^{\circ}$ & $0.72^{*}$ & 0.19 & $0.63^{\prime \prime}$ & $0.59^{\circ}$ & 0.07 \\
\hline 6 & -0.25 & 0.31 & $0.38^{\circ}$ & -0.06 & 0.09 & 1 & -0.15 & 0.04 & -0.29 & $0.37^{\circ}$ & $0.39^{\circ}$ & -0.1 & 0.12 & $0.43^{\circ}$ & 0.1 \\
\hline 7 & -0.26 & 0.28 & 0.35 & -0.16 & $0.45^{\circ}$ & 0.04 & 1 & -0.31 & -0.28 & 0.25 & 0.25 & -0.005 & 0.32 & 0.26 & 0.1 \\
\hline 8 & $0.53^{\prime \prime}$ & -0.2 & $-0.39^{\circ}$ & 0.11 & $-0.41^{\circ}$ & -0.17 & -0.22 & 1 & $0.58^{*}$ & -0.23 & -0.18 & -0.28 & -0.22 & -0.18 & -0.16 \\
\hline 9 & 0.15 & -0.1 & -0.18 & 0.2 & -0.14 & -0.14 & -0.13 & $0.52^{*}$ & 1 & -.015 & -0.17 & 0.07 & -0.13 & $-0.37^{\circ}$ & -0.24 \\
\hline 10 & -0.32 & $0.6^{\circ}$ & $0.83^{*}$ & 0.33 & $0.58^{*}$ & $0.41^{\circ}$ & $0.44^{\circ}$ & -0.27 & -0.14 & 1 & $0.97^{\prime \prime}$ & 0.09 & $0.62^{\prime \prime}$ & $0.78^{* *}$ & 0.16 \\
\hline 11 & -0.33 & $0.53^{\prime \prime}$ & $0.8^{* \prime}$ & 0.32 & $0.6^{\circ}$ & $0.37^{\circ}$ & $0.43^{\circ}$ & -0.28 & -0.19 & $0.94^{\circ}$ & 1 & -0.1 & $0.63^{\prime \prime}$ & $0.81^{*}$ & 0.26 \\
\hline 12 & -0.21 & $0.5^{\prime \prime}$ & $0.54^{\circ}$ & 0.18 & 0.3 & 0.28 & 0.35 & -0.12 & 0.01 & $0.68^{* *}$ & $0.41^{\circ}$ & 1 & -0.04 & -0.14 & $-0.49^{*}$ \\
\hline 13 & -0.3 & $0.41^{\circ}$ & $0.55^{\circ}$ & 0.07 & $0.58^{*}$ & $0.4^{\circ}$ & $0.71^{*}$ & -0.26 & -0.27 & $0.7^{* \prime}$ & $0.65^{\circ}$ & $0.55^{\circ}$ & 1 & $0.61^{*}$ & -0.004 \\
\hline 14 & $-0.44^{\circ}$ & $0.45^{\circ}$ & $0.64^{*}$ & $0.37^{\circ}$ & $0.87^{*}$ & 0.26 & $0.38^{\circ}$ & $-0.55^{\circ}$ & -0.23 & $0.69^{*}$ & $0.68^{*}$ & $0.42^{\circ}$ & $0.57^{\circ}$ & 1 & $0.42^{\circ}$ \\
\hline 15 & $-0.38^{\circ}$ & 0.22 & 0.13 & 0.12 & 0.34 & -0.01 & $0.55^{\circ}$ & -0.35 & $-0.42^{\circ}$ & 0.26 & 0.22 & 0.29 & $0.42^{\circ}$ & $0.38^{\circ}$ & 1 \\
\hline
\end{tabular}

1-Plant height; 2-Panicle length; 3-Number of panicles per plant; 4-Flag leaf length; 5-Flag leaf width; 6-Brown grain length; 7-Brown grain width; 8-Days to 50\% flowering; 9-Days to maturity; 10- Number of filled grains per panicle,;11-Number of spikelet per panicle; 12-Panicle fertility percentage; 13-1000-Grain weight; 14-Paddy yield; 15-Chlorophyll index

Tab. 6. Genotypic correlation coefficients between traits in 30 rice cultivars under two irrigation regimes, optimum irrigation (up) and drought stress (down)

\begin{tabular}{|c|c|c|c|c|c|c|c|c|c|c|c|c|c|c|c|}
\hline Trait & 1 & 2 & 3 & 4 & 5 & 6 & 7 & 8 & 9 & 10 & 11 & 12 & 13 & 14 & 15 \\
\hline 1 & 1 & -0.14 & $-0.72^{\prime \prime}$ & $0.33^{\circ}$ & -0.18 & $-0.44^{\circ}$ & 0 & 0.15 & 0.31 & $-0.51^{\prime \prime}$ & $-0.61^{*}$ & $0.5^{\circ}$ & -0.31 & $-0.75^{\prime \prime}$ & -0.72 \\
\hline 2 & -0.13 & 1 & $0.51^{*}$ & 0.18 & $0.55^{\circ}$ & 0.23 & 0 & -0.22 & -0.2 & $0.59^{*}$ & $0.56^{\circ}$ & 0.15 & $0.43^{\circ}$ & 0.5 & -0.3 \\
\hline 3 & $0.47^{\prime \prime}$ & $0.64^{\circ}$ & 1 & 0.03 & $0.43^{\circ}$ & $0.47^{\circ}$ & 0 & -0.25 & -0.32 & $0.64^{*}$ & $0.68^{* \prime}$ & -0.2 & $0.49^{\prime \prime}$ & $0.79^{*}$ & $0.37^{\circ}$ \\
\hline 4 & 0.11 & 0.23 & 0.27 & 1 & 0.23 & -0.04 & 0 & $0.34^{\circ}$ & 10.18 & 0.04 & 0.03 & 0.06 & 0.32 & 0.2 & -0.22 \\
\hline 5 & -0.28 & $0.5^{\prime \prime}$ & $0.57^{\circ}$ & $0.41^{\circ}$ & 1 & 0.06 & 0 & -0.29 & -0.09 & $0.81^{*}$ & $0.76^{\circ}$ & 0.23 & $0.72^{*}$ & $0.67^{*}$ & 0.11 \\
\hline 6 & -0.26 & 0.32 & $0.39^{\circ}$ & -0.07 & 0.07 & 1 & 0 & 0.05 & -0.31 & $0.37^{\circ}$ & $0.39^{\circ}$ & -0.11 & 0.14 & $0.49^{\circ}$ & 0.11 \\
\hline 7 & -0.28 & 0.3 & 0.37 & -0.18 & $0.45^{\circ}$ & 0 & 1 & 0 & 0 & 0 & 0 & 0 & 0 & 0 & 0 \\
\hline 8 & $0.58^{\prime \prime}$ & -0.22 & $-0.43^{\circ}$ & 0.13 & $-0.53^{*}$ & -0.18 & -0.27 & 1 & $0.64^{\circ}$ & -0.24 & -0.19 & -0.3 & -0.24 & -0.23 & -0.18 \\
\hline 9 & 0.17 & -0.11 & -0.19 & 0.23 & -0.19 & -0.16 & -0.15 & $0.62^{\prime \prime}$ & 1 & -0.17 & -0.19 & 0.08 & -0.13 & $-0.44^{\circ}$ & -0.32 \\
\hline 10 & -0.33 & $0.61^{\prime \prime}$ & $0.84^{\prime \prime}$ & 0.35 & $0.64^{\circ}$ & $0.41^{\circ}$ & $0.46^{\circ}$ & -0.29 & -0.16 & 1 & $0.98^{*}$ & 0.09 & $0.7^{*}$ & $0.89^{\circ}$ & 0.17 \\
\hline 11 & -0.33 & $0.54^{\circ}$ & $0.81^{*}$ & 0.34 & $0.66^{\circ}$ & $0.38^{\circ}$ & $0.45^{\circ}$ & -0.31 & -0.21 & $0.95^{\circ}$ & 1 & -0.11 & $0.7^{*}$ & $0.92^{*}$ & 0.3 \\
\hline 12 & -0.22 & $0.52^{*}$ & $0.56^{\circ}$ & 0.19 & 0.33 & 0.29 & $0.37^{\circ}$ & -0.12 & 00.01 & $0.69^{*}$ & $0.43^{\circ}$ & 1 & -0.02 & -0.15 & $-0.62 "$ \\
\hline 13 & $-0.35^{\circ}$ & $0.48^{\prime \prime}$ & $0.62^{*}$ & 0.05 & $0.77^{\circ}$ & $0.46^{\circ}$ & $0.84^{\circ}$ & -0.33 & $-0.33^{\circ}$ & $0.81^{*}$ & $0.75^{\prime \prime}$ & $0.67^{\circ}$ & 1 & $0.78^{\prime \prime}$ & -0.01 \\
\hline 14 & $-0.47^{*}$ & $0.49^{\prime \prime}$ & $0.7^{* \prime}$ & $0.41^{\circ}$ & $1.02^{*}$ & 0.29 & $0.42^{\circ}$ & $-0.65^{*}$ & -0.31 & $0.75^{*}$ & $0.73^{*}$ & $0.47^{\circ}$ & $0.71^{*}$ & 1 & $0.55^{\circ}$ \\
\hline 15 & $-0.52^{*}$ & 0.31 & 0.19 & 0.18 & $0.6^{*}$ & -0.02 & $0.81^{*}$ & $-0.52^{*}$ & $-0.58^{*}$ & $0.35^{\circ}$ & 0.3 & $0.39^{\circ}$ & $0.66^{\circ}$ & $0.6^{\prime \prime}$ & 1 \\
\hline
\end{tabular}

1-Plant height; 2-Panicle length; 3-Number of panicles per plant; 4-Flag leaf length; 5-Flag leaf width; 6-Brown grain length; 7-Brown grain width; 8-Days to 50\% flowering; 9-Days to maturity; 10- Number of filled grains per panicle,;11-Number of spikelet per panicle; 12-Panicle fertility percentage; 13-1000-Grain weight; 14-Paddy yield; 15-Chlorophyll index

otype correlation under drought stress conditions showed that paddy yield had significant and positive correlation with panicle length, panicles number per plant, flag leaf width, number of filled grains per panicle, number of spikelet per panicle, panicle fertility percentage, 1000grain weight and chlorophyll index but it had significant and negative correlation with plant height and number of days to $50 \%$ flowering (Tab. 6). Path analysis results under optimum irrigation regime condition showed that, num- ber of spikelet per panicles had the most direct positive and direct effect $(0.55)$ on paddy yield. Indirect effect of these traits had 0.27 approximately; therefore due to days to complete maturity (0.04), flag leaf length (0.01) and plant height (0.22) were effective on paddy yield (Tab. 7). The days to complete maturity had a negative direct effect $(-0.22)$ on yield after spikelet number per panicle and indirect effect of this trait was approximately -0.14 and through respectively number of spikelet per panicle (-0.09), flag 
Tab. 7. Path analysis for paddy yield of 30 studied rice varieties under optimum irrigation regime. The bold values are direct effects (path coefficients)

\begin{tabular}{lcccccc}
\hline \multicolumn{1}{c}{ Traits } & $\begin{array}{c}\text { Number of spikelet } \\
\text { per panicle }\end{array}$ & $\begin{array}{c}\text { Days to complete } \\
\text { maturity }\end{array}$ & $\begin{array}{c}\text { Flag leaf } \\
\text { length }(\mathrm{cm})\end{array}$ & $\begin{array}{c}\text { Plant height } \\
(\mathrm{cm})\end{array}$ & $\begin{array}{c}\text { Total indirect } \\
\text { effects }\end{array}$ & $\begin{array}{c}\text { Correlation with } \\
\text { Paddy yield }\end{array}$ \\
\hline Number of spikelet per panicle & $\mathbf{0 . 5 5}$ & 0.04 & 0.01 & 0.22 & 0.27 & 0.82 \\
Days to complete maturity & -0.09 & $\mathbf{- 0 . 2 2}$ & 0.05 & -0.10 & -0.14 & -0.36 \\
Flag leaf length $(\mathrm{cm})$ & 0.02 & -0.04 & $\mathbf{0 . 3 1 7}$ & -0.12 & -0.14 & 0.17 \\
Plant height & -0.33 & -0.06 & 0.10 & $-\mathbf{0 . 3 6}$ & -0.29 & $-0.65^{* *}$ \\
& \multicolumn{2}{c}{$\mathrm{R}^{2}=0.827$} & & & \\
\hline
\end{tabular}

${ }^{*}$ and ${ }^{* *}$ : significant at $5 \%$ and $1 \%$ probability levels, respectively

Tab. 8. Path analysis for paddy yield of 30 studied rice varieties under drought stress condition. The bold values are direct effects (path coefficients)

\begin{tabular}{lccccc}
\hline \multicolumn{1}{c}{ Traits } & $\begin{array}{c}\text { Flag leaf } \\
\text { width }(\mathrm{cm})\end{array}$ & $\begin{array}{c}\text { Number of filled } \\
\text { grains per panicle }\end{array}$ & $\begin{array}{c}\text { Days to 50\% } \\
\text { flowering }\end{array}$ & $\begin{array}{c}\text { Total indirect } \\
\text { effects }\end{array}$ & $\begin{array}{c}\text { Correlation with } \\
\text { Paddy yield }\end{array}$ \\
\hline Flag leaf width $(\mathrm{cm})$ & $\mathbf{0 . 6 3}$ & 0.16 & 0.09 & 0.25 & $0.88^{*}$ \\
Number of filled grains per panicle & 0.36 & $\mathbf{0 . 2 7}$ & 0.06 & 0.42 & $0.69^{*}$ \\
Days to 50\% flowering & -0.26 & -0.07 & $\mathbf{- 0 . 2 2}$ & -0.33 & $-0.55^{* *}$ \\
& \multicolumn{2}{c}{$\mathrm{R}^{2}=0.861$} & & \\
\hline
\end{tabular}

${ }^{*}$ and ${ }^{* *}$ : significant at $5 \%$ and $1 \%$ probability levels, respectively.

leaf length $(0.05)$ and plant height $(-0.1)$ on paddy yield. Flag leaf length had a positive and direct effect $(0.317)$ on paddy yield and its indirect effects were through traits including number of spikelet per panicle, days to complete maturity and plant height, $0.02,-0.04$ and -0.12 , respectively, on paddy yield. Last trait into the path analysis model was plant height with a direct effect $(-0.36)$ on paddy yield and negative indirect effect on paddy yield was through both the number of spikelet per panicle -0.33 , days to complete maturity -0.06 and flag leaf length 0.1 . The number of spikelets per panicle were the most important trait effective on paddy yield and it may be suitable for selective and breeding. Determination of the most important selection scale for breeding of paddy yield is different, but in most of researches yield components have been reported, as an important criterion. In many researches panicle number per plant (Paul and Nanda, 1994; Yadav and Bhushan, 2001) and also number of spikelet per panicle (Yolanda and Das, 1995; Zheng et al., 2003) have been reported as effective traits with the highest direct effect on grain yield to improve of grain yield breeding. Basavaraja et al. (1997) studied 10 traits related to yield components on $\mathrm{F}_{2}$ cultivars and they concluded that, panicles number per plant have the highest positive direct effect on grain yield. In similar studies of Padmavathi et al. (1996) perfuming of path analysis on 23 rice cultivars showed that, indirect selection based on panicle number per plant and panicle length can be useful tool for increasing grain yield. Under drought stress conditions flag leaf width had the highestpositive and direct effect on paddy yield (0.62). Indirect effects of flag leaf width on yield were due to number of filled grains per panicle (0.16) and days to $50 \%$ flowering (0.09) and total 0.25. After this trait, number of filled grain per panicle (0.27) and days to $50 \%$ flowering $(-0.22)$ had the highest direct effect on yield, respectively (Tab. 8). Number of filled grains per panicle had a direct and positive effect $(0.27)$ and also a positive and indirect effect was observed via flag leaf width and days to $50 \%$ flowering on yield that is 0.36 and 0.06 respectively. Days to $50 \%$ flowering had negative and direct effect $(-0.22)$ and negative indirect effects via flag leaf width and number of filled grains per panicle were -0.26 and -0.07 , respectively. Similarly path coefficients was observed so that the largest direct effect showed a significant difference between the direct effects and other traits were belonging to flag leaf width. In addition, number of filled grains per panicle and days to $50 \%$ flowering had significant and indirect effect on paddy yield through the flag leaf width. So it seems that, the flag leaf width was the most important influencing trait on paddy yield and it will be studied as good selection criteria in order to yield breeding programs. Mehetre et al. (1994) have been reported that, number of filled grain per panicle, plant height and panicle length are the most important and effective traits on yield for breeding of upland rice.

\section{Conclusions}

The results of this research showed that indirect selections for increasing the number of spikelet per panicle and flag leaf length and decreasing plant height and days to complete maturity under optimum irrigation, and for increasing flag leaf width and number of filled grains per panicle and reducing days to $50 \%$ flowering can be suitable to improve paddy yield of rice in breeding programs. 
142

\section{Acknowledgment}

This research was supported by funding from the University of Guilan, Rasht, Iran.

\section{References}

Abd El Samie FS, Hassan RK (1994). Correlation and path analysis of some yield attributes in rice varieties. Ann Agricult Sci Moshtohor 3:1157-1166.

Bapo JRK, Soundarapandian G (1992). Genotypic association and path analysis in $\mathrm{F}_{3}$ generation of rice crosses. Madras Agric J 11:619-623.

Basavaraja P, Rudradhya M, Kulkarni RS (1997). Genetic variability, correlation and path analysis of yield components in two $\mathrm{F}_{4}$ population of fine grained rice. Mysore J Agricult Sci 1:1-6.

Botwright Acuna TL, Lafitte HR, Wade LJ (2008). Genotype and environment interactions for grain yield of upland rice backcross lines in diverse hydrological environments. Field Crops Res 108(2):117-125.

Choudhury PKD, Das PK (1998). Genetic variability, correlation and path coefficient analysis in deep water rice. Ann Agricult Res 2:120-132.

Dewey DR, Lu KH (1959). A correlation and path coefficient analysis of components of crested wheat grass seed production. Agron J 51:515-518.

Ekanayake IJ, Garrity DP, Masajo TM, O’Toole JC (1985). Root pulling resistance in rice: inheritance and association with drought tolerance. Euphytica 34(3):905-913.

Garrity DP, O’Toole JC (1994). Screening rice for drought resistance at the reproductive phase. Field Crop Res 39(23):99-100.

Jearakongman S, Rajatasereekul S, Naklang K, Romyen P, Fukai S, Skulkh E, Jumpaket UB, Nathabutr K (1995). Growth and grain yield of contrasting rice cultivars grown under different conditions of water availability. Field Crop Res 44(2-3):139-150.

Jun L, Dokawa T, Hirasawa T (2000). The effects of irrigation regimes on the water use, dry matter production and physiological responses of paddy rice. Plant Soil 223:207216.

Lafitte R, Blum A, Altin G (2003). Using secondary traits to help identify drought tolerant genotypes. In: Fischer KS, Lafitte R, Fukai S, Altin G, Hardy B (Eds.). Breeding rice for drought-prone environment. International Rice Research Institute. Los Banos Philippines.
Lafitte HR, Vijayakumar ZKLi CHM, Gao YM, Shi Y, Xu JL, Fu BY, Yu SB, Ali AJ, Domingo J, Maghirang R, Torres R, Mackill D (2006). Improvement of rice drought tolerance through backross breeding: Evalution of donors and selection in drought nurseries. Field Crops Res 97:77-86.

Lanceras JC, Griengrai P, Boonrat J, Theerayut T (2004). Quantitative trait loci associated with drought tolerance at reproductive stage in rice. Plant Physiol 1:84-399.

Kumar R, Sarawgi AK, Ramos C, Amarante ST, Ismail AM, Wade LJ (2006). Partitioning of dry matter during drought stress in rainfed lowland rice. Field Crops Res 98(1):1-11.

Kumar R, Venuprasad R, Altin GN (2007). Genetic analysis of rainfed lowland rice drought tolerance under naturallyoccurring stress in eastern India: Heritability and QTL effects. Field Crops Research 103(1):42-52.

Mehetre SS, Mahajan CR, Patil PA, Land SK, Dhumal PM (1994). Variability, heritability, correlation, path analysis and genetic divergence studies in upland rice. IRRI Note 19(1):8-10.

Padmavathi N, Mahadevappa M, Reddy OUK (1996). Association of various yield components in rice (Oryza sativa L.). Crop Res Hisar 12:353-357.

Paul CR, Nanda JS (1994). Path analysis of yield and yield components and construction of selection indices of direct seeded rice: first season. Annual review conference proceedings. National Agriculture Research Institute, Caribbean Agricultural Research and Development Institute, Guyana, 63-71 p.

Singh KA (2003). Enhancing rice productivity in water stressed environments. IRRI Publications DOI No: 10.1142/9789814280013_0013.

Widawsky DA, O'Toole JC (1990). Prioritizing the rice biotechnology research agenda for Eastern India. New York, NY, The Rockefeller Foundation.

Yadav RS, Bhushan C (2001). Effect of moisture stress on growth and yield in rice genotypes. Ind J Agricult Res 2:104-107.

Yang J, Zhang J (2006). Grain filling of cereals under soil drying. New Phytol 169(2):223-236.

Yolanda JL, Das LDV (1995). Correlation and path analysis in rice (Oryza sativa L.). Madras Agricult J 11:576-578.

ZhengJG, Ren GJ, Lu XJ, Jiang XL (2003). Effect of water stress on rice grain yield and quality after heading stage. Chin $\mathrm{J}$ Rice Sci 3:239-243. 